\title{
RULE-BASED CLASSIFICATION OF A HYPERSPECTRAL IMAGE USING MSSC HIERARCHICAL SEGMENTATION
}

\author{
D. Akbari ${ }^{\mathrm{a}, *}$, A.R. Safari ${ }^{\mathrm{a}}$ \\ ${ }^{a}$ Surveying and Geomatics Engineering Department, \\ College of Engineering, University of Tehran, Tehran, Iran - \\ (davoodakbari, asafari) @ut.ac.ir
}

KEY WORDS: hyperspectral image, Rule-based Classification, hierarchical segmentation, marker selection, Feature Extraction

\begin{abstract}
:
The Hierarchical SEGmentation (HSEG) algorithm, which combines region object finding with region object clustering, has given good performances for hyperspectral image analysis. This technique produces at its output a hierarchical set of image segmentations. The automated selection of a single segmentation level is often necessary. In this paper, we propose to use spectral-spatial classifiers at the marker selection procedure, each of them combining the results of a pixel-wise classification and a segmentation map. Then, a novel marker-based HSEG algorithm (that is called Multiple Spectral-Spatial Classifier-HSEG (MSSC-HSEG)) is applied, resulting in a segmentation map. The segmentation results are then used in a rule-based classification using spectral, geometric, textural, and contextual information. The experimental results, presented for a hyperspectral airborne image, demonstrate that the proposed approach yields accurate segmentation and classification maps, when compared to previously classification techniques.
\end{abstract}

\section{INTRODUCTION}

Imaging spectroscopy (Goetz, 1985), also known as hyperspectral (HS) imaging, is concerned with the measurement, analysis, and interpretation of spectra acquired from a given scene (or specific object) at a short, medium or long distance by an airborne or satellite sensor. The concept of imaging spectroscopy originated in the 1980's, when A. F. H. Goetz and his colleagues at NASA's Jet Propulsion Laboratory began a revolution in remote sensing by developing new instruments such as the Airborne Imaging Spectrometer (AIS), then called AVIRIS, for Airborne Visible Infra-Red Imaging Spectrometer (Green, 1998). This system is now able to cover the wavelength region from 0.4 to $2.5 \mu \mathrm{m}$ using more than two hundred spectral channels, at nominal spectral resolution of 10 nm.

The recent developments in spatial, spectral and radiometric resolution have stimulated new methodologies for land cover and land use classification. An extensive literature is available on the classification of HS images. Among them, one can separate pixel-wise processing techniques that work on the spectral information only (one of the most frequently used techniques are Support Vector Machines (SVM) (Camps-Valls, 2005a)) and spectral-spatial classification techniques that take into consideration both the spectra of the pixels and their spatial context (Fauvel, 2008). The importance of analyzing spatial and spectral patterns simultaneously has been identified as a desired goal by many scientists devoted to multidimensional data analysis. This type of processing has been approached in the past from various points of view. For instance, several possibilities are discussed in (Landgrebe, 2003) for the refinement of results obtained by spectral-based techniques in multispectral imaging through a second step based on spatial context. Such contextual classification, extended also to HS images (Jimenez, 2005), accounts for the tendency of certain ground cover classes to occur more frequently in some contexts than in others.
In the seminal works on spectral-spatial image classification, the information from the closest neighborhoods, defined by either fixed windows (Kettig, 1976 and Camps-Valls, 2006b) or morphological profiles (Fauvel, 2008), has been considered for classifying each pixel. In recent works, it has shown the advantage of using segmentation for distinguishing spatial structures in a HS image (Tarabalka, 2010c and Tarabalka, 2010b). Different unsupervised techniques have been investigated for segmentation of HS data, such as watershed, partitional clustering, and Hierarchical SEGmentation (HSEG). The HSEG method is one of the few available segmentation approaches in the state-of-art that naturally integrates spatial and spectral information. HSEG is a combination of region object finding by hierarchical step-wise optimization (HSWO, or iterative best merge region growing) (Beaulieu, 1989) and region clustering by grouping spectrally similar but spatially disjoint regions (Tilton, 1998a and Tilton 2010d).

An alternative way to get accurate segmentation results consists in performing a marker-controlled segmentation (Gonzalez, 2002 and Soille 2003). The idea behind this approach is to select, for every spatial object, one or several pixels belonging to this object (called a region seed, or a marker of the corresponding region) and to grow regions from the selected seeds so that every region in the resulting segmentation map is associated with one region seed. Tarabalka et al. have proposed to use probability estimates obtained by the pixel-wise support vector machine (SVM) classification in order to select the most reliable classified pixels as markers, i.e., seeds of spatial regions. Furthermore, a Minimum Spanning Forest (MSF) rooted on the selected markers was constructed, resulting in a segmentation and classification map. The drawback of this method is that the choice of markers strongly depends on the performances of the selected pixel-wise classifier. For this purpose, several individual classifiers must be chosen and combined within one system in such a way that the complementary benefits of each classifier are used, while their weaknesses are avoided. Therefore we have used two spectralspatial classifiers independently to classify an image, each of them combining the results of a pixel-wise classification and 
one of the unsupervised segmentation techniques. Then a marker map is constructed by selecting the pixels assigned by two the classifiers to the same class.

To differentiate impervious urban land covers such as buildings, roads, and shadow and bare soil additional information should be incorporated into the classification process. Additional information could be the spatial measures extracted either from the image, in the forms of textural, morphological, and contextual measures, or from ancillary data (Salehi, 2012). The attributes most frequently used are spectral and textural parameters, area, perimeter, and compactness (Carleer, 2006, Shackelford, 2003 and Jensen, 2004). In this paper we propose a new Marker-based HSEG (MSSC-HSEG) method for segmentation of HS images. Then, the segmented image is used in a rule-based classification method that considers spectral, geometric, and contextual information.

Experimental results are demonstrated on a HS airborne image acquired by the Reflective Optics System Imaging Spectrometer (ROSIS). The proposed approach is compared with two other spectral-spatial classification techniques. The outline of this paper is as follows. The next section describes proposed Classification scheme. In Section III, Experimental results are discussed and conclusions are drawn in Section IV.

\section{PROPOSED CLASSIFICATION SCHEME}

The flowchart of the proposed classification scheme is shown in Fig. 1. So that Notice, the proposed approach contains four main sections, Marker Selection, Marker-based HSEG, Feature Extraction and rule-based classification, we describe them in continuance.

\section{A. Marker Selection}

This step consists in computing a map of markers, using classification maps and exclusionary rule: For every pixel, if all the classifiers agree, the pixel is kept as a marker, with the corresponding class label. The resulting map of markers contains the most reliable classified pixels.

Therefore, two classifiers are used independently to classify an image. Consequently, each of them combining the results of a pixel-wise classification and a segmentation map using the Majority Voting (MV) principle. Used segmentation methods are watershed and Expectation Maximization. In the following, two using segmentation algorithms, pixel-wise classification and majority voting principle is described.
1) Watershed segmentation: Watershed transformation is a powerful morphological approach to image segmentation which combines region growing and edge detection (Tarabalka, 2008e). The watershed is usually applied to the gradient function, and it divides an image into regions, so that each region is associated with one minimum of the gradient image. Tarabalka et al. have extended a watershed method to the case of HS images: First, a one-band Robust Color Morphological Gradient (RCMG) for the HS image is computed. By applying watershed transformation using a classical algorithm (Vincent, 1991), the image is partitioned into a set of regions.

2) Segmentation by expectation maximization: The Expectation Maximization (EM) algorithm for the Gaussian mixture resolving belongs to the group of partitional clustering techniques (Tarabalka, 2009a). Clustering aims at finding groups of spectrally similar pixels. We assume that pixels belonging to the same cluster are drawn from a multivariate Gaussian probability distribution. The parameters of the distributions are estimated by the EM algorithm. When the algorithm converges, the partitioning of the set of image pixels into clusters is obtained. However, as no spatial information is used during the clustering procedure, pixels with the same cluster label can form a connected spatial region, or can belong to disjoint regions. In order to obtain segmentation map, a connected components labeling algorithm is applied to the output image partitioning obtained by clustering.

3) Pixel-wise classification: Independently of the previous steps, a pixel-wise classification of the HS image is performed. We propose to use an SVM classifier for this purpose which is well suited for classifying HS data (Camps-Valls, 2005a).

4) Majority voting within segmentation regions: Each of the obtained segmentation maps is combined with the pixel-wise classification map using the majority voting principle: For every region in the segmentation map, all the pixels are assigned to the most frequent class within this region.

\section{B. Marker-based HSEG}

The HSEG algorithm is a segmentation technique based on iterative hierarchical stepwise optimization (HSWO) regiongrowing method. Furthermore, it provides a possibility of merging nonadjacent regions by spectral clustering (Tilton, 2008c). The following outline of the HSEG algorithm is based on the description given in (Tilton, 2008c) and (Tilton, 2003b) which can be summarized in three steps:

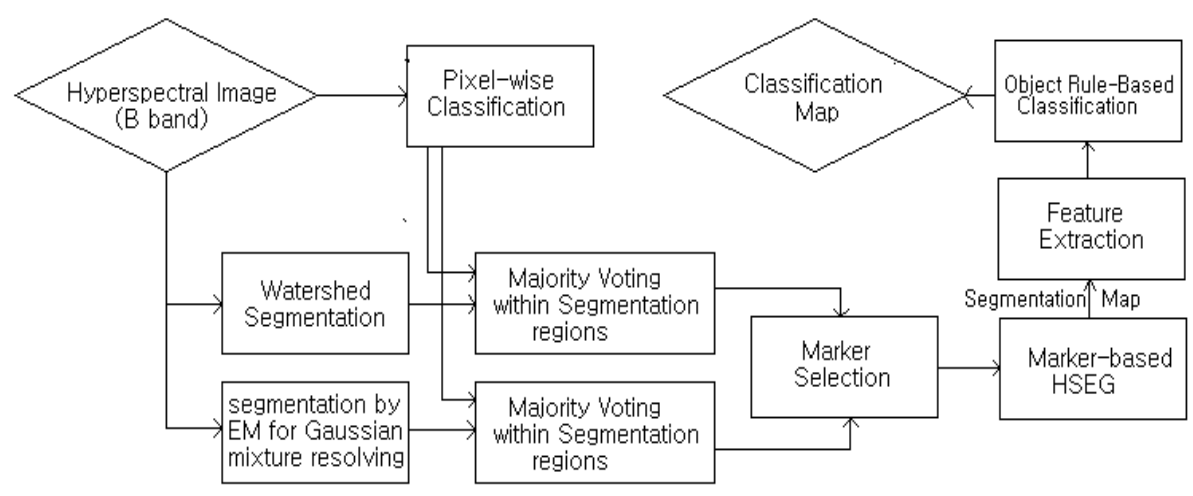

Figure 1. Flow-chart of the proposed classification scheme. 
1) Initialize the segmentation by assigning for each pixel a region label. If a pre-segmentation is provided, label each pixel according to the presegmentation. Otherwise, label each pixel as a separate region.

2) Calculate the dissimilarity criterion value between all pairs of spatially adjacent regions, find the pair of spatially adjacent regions with the smallest dissimilarity criterion value, and merge that pair of regions.

3) Stop if no more merges are required. Otherwise, return to step 2).

When determining most similar pair of regions, we propose to choose the standard Spectral Angle Mapper (SAM) between the region mean vectors and as a dissimilarity criterion (Tilton, 2008c). The SAM measure between $u_{i}$ and $u_{j}\left(u_{i}, u_{j}\right.$ $\in R^{B}$ ) determines the spectral similarity between two vectors by computing the angle between them. It is defined as:

$$
\operatorname{SAM}\left(u_{i}, u_{j}\right)=\arccos \left(\frac{\sum_{b=1}^{B} u_{i b} u_{j b}}{\left[\sum_{b=1}^{B} u_{i b}^{2}\right]^{1 / 2}\left[\sum_{b=1}^{B} u_{j b}^{2}\right]^{1 / 2}}\right)
$$

HSEG algorithm allows merging of non-adjacent regions using a controlling parameter $S_{w g h t}$ input parameter. The optional parameter $S_{w g h t}$ tunes the relative importance of spectral clustering versus region growing. For $S_{w g h t}=0.0$, HSEG is essentially the same as HSWO where only the spatially adjacent regions are allowed to merge. For $S_{w g h t}=1.0$, the spatially adjacent and non-adjacent regions are given the equal weights for merging. Finally, for the values of $S_{w g h t}$ between 0.0 and 1.0 , spatially adjacent merges are favored compared with spatially nonadjacent Merges by a factor of $1.0 / S_{w g h t}$. The main idea behind the marker-based HSEG algorithm consists in assigning a marker label for each region containing marker pixels, and merging regions with an additional condition: two regions with different marker labels cannot be merged together. The proposed MSSC-HSEG algorithm can be summarized as follows:

First initializes each pixel as one region. If the given pixel is marked, the corresponding region obtains a new non-zero marker label, with the corresponding information class. Thus, at the initialization step all the markers are split into one-pixel markers. Then, iterative region merging is performed, providing that regions with different markers cannot be merged together. At the final step, the regions containing pixels of the same initial marker are merged together.

\section{Feature Extraction}

Because of the complex nature and diverse composition of land cover types found within the urban environment, classification of high-resolution HS satellite imagery is a difficult task. For example, the Grass and Tree classes in the image are spectrally similar and have a significant amount of spectral overlap. This is the primary reason for the large number of misclassifications between these classes. Traditional classification methods that only take into account spectral information are unable to differentiate between these classes with a high degree of accuracy. Consequently, methods that utilize spatial information in addition to spectral information are needed to produce more accurate land cover classifications of high resolution image data over urban areas. The goal of this paper is use the classification techniques for extraction of urban area geospatial information produced from HS satellite imagery.

There is plenty of geometrical information such as object feature, shape feature, texture, and contextual relation feature and so on. And the feature of Rule-based classification is object feature from segmented image. The spatial information extracted from objects can help to decrease the number of Misclassifications between the spectrally similar classes However, while one spatial feature might increase the classification accuracy between one set of classes, it might decrease the accuracy between another set using traditional classification methods. So that different classes should only be classified using the spatial measures best suited for those classes. In this paper we add other feature information into feature space, which are area, entropy, shape index and contextual relation feature.

1) Area feature: The area of an image object is the number of pixels forming it. While the Grass and Tree classes can have similar spectral signatures, areas in the image covered with Grass larger than Tree covered areas.

2) Entropy feature: With the Grass and Tree classes, areas in the image covered with grass appear much more homogeneous than tree-covered areas. This difference in homogeneity between regions can be used to decrease the confusion between the classes. Several of the other statistical texture measures show increase in the accuracy of these classes, but not as large as the increase found when using the entropy measure. Entropy texture measure is calculated as follows:

$$
\text { entropy }=-\sum_{i=0}^{L-1} p\left(z_{i}\right) \log _{2} p\left(z_{i}\right)
$$

Where $\mathrm{L}$ is the number of distinct gray levels, $\mathrm{z}$ is a random variable denoting image gray levels and $p\left(z_{i}\right)$ is the normalized gray level histogram.

3) Shape feature: We use shape feature as follows ( $L i, 2007)$ :

$$
L=\sqrt{S} / P
$$

In this formula, $\mathrm{S}$ is the area of a certain polygon object and $\mathrm{P}$ is the perimeter. $L$ is called shape index of object. Shape index of rectangle or square is bigger than linear object. Spectrally similar between roads and buildings in urban space, we can distinguish each other by shape index.

4) Adjacency feature: The adjacency feature is intelligent understanding to the image. For instant, the objects of buildings and roads have the same object features, and we cannot distinguish these types commendably. But there exist visible 
shadow objects in some direction around high buildings, so we can distinguish these two objects by the adjacency information.

5) Relation feature: We define relation feature as follows: if object $\mathrm{A}$ and $\mathrm{B}$ are two adjacent objects and $\mathrm{A}$ and $\mathrm{B}$ are the same class then $\mathrm{A}$ has relation with $\mathrm{B}$. If $\mathrm{B}$ has relation with $\mathrm{C}$ and $\mathrm{C}$ is not adjacent with $\mathrm{A}$ then $\mathrm{A}$ has relation with $\mathrm{C}$. Relation feature is the number of objects has relation with A.

\section{Rule-Based Classification}

The rule-based approach allows the analyst to combine different features of objects in order to assign a class membership degree (between 0 and 1) to each object based on a fuzzy membership function or strict thresholds (Benz, 2004 and Walker, 2008). The membership functions used in this study are based on the logical operator AND $(\&)$ and thresholds. Furthermore, it has a hierarchical capability to classify the entire scene into general classes (e.g., vegetation and non-vegetation areas). These general classes are called parent classes. Then, each parent class is divided to sub classes (child class) containing more detailed land cover types (e.g., buildings and roads). This hierarchical capability allows the developer to incorporate objects in different levels of segmentation for individual levels of class hierarchy. In this paper, we developed a rule-based classification scheme that allows the image to be hierarchically classified using different spatial measures for different sets of classes.

Several rules can be applied for a segment. The more rules are satisfied for a segment, the more likely it is that the result is accurate. This provides a means for increasing or decreasing classification certainty while combining knowledge contained in the rules. Also, the quality results of a rule-based system depend on rule weighting, since they do not have the same importance. The importance of each rule can change according to the application's context. There is no automatic method to determine rule weighting (Voirin, 2004). Thus, the user must define a weight for every rule based on his experience and knowledge of the environment. In this study, we propose a semiautomatic technique to help the user determine the rule weighting. The technique works as follows: The classification rules are applied separately, and the results are compared to the reference data. This yields the identification accuracy for each rule, which is the proposed rule weight.

\section{EXPERIMENTAL ANALYSIS}

The experimental analysis was carried out on a HS image acquired over the city center of Pavia (Italy) by the ROSIS-03 (Reflective Optics Systems Imaging Spectrometer) HS sensor. The image has a geometrical resolution of $1.3 \mathrm{~m}$ and $785 \times 300$ pixels. The original data are composed of 115 spectral bands, ranging from 0.43 to $0.86 \mu \mathrm{m}$ with a band of $4 \mathrm{~nm}$. However, noisy bands were previously discarded, leading to 102 channels. The thematic classes found were Water, Tree, Meadow, Selfblocking Bricks, Soil, Asphalt, Bitumen, Tile, and Shadow. The training and test sets were composed of 4536 and 53539 samples, respectively. The training set was used for training an SVM classifier, while the test set was employed for estimating classification accuracy. The true color representation of the image and reference data is shown in Fig. 2.

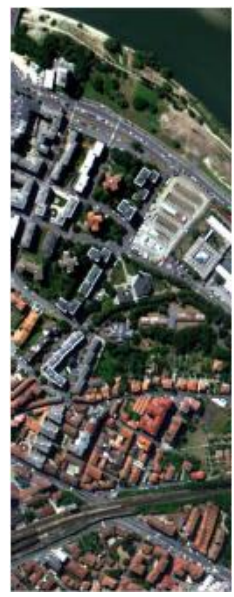

(a)

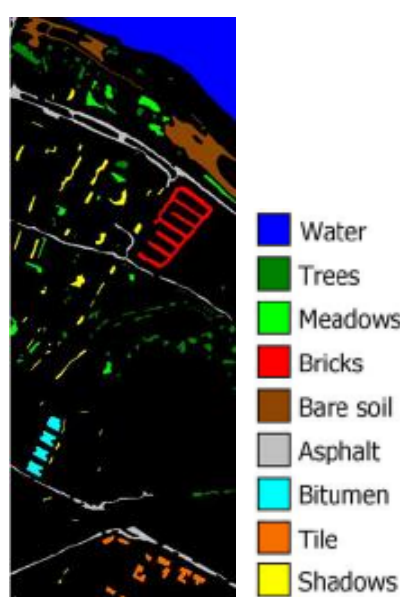

(b)
Figure 2. ROSIS Pavia data sets: (a) True color representation and (b) Reference set

First, marker selection was performed, using two different Segmentation techniques discussed in the previous section. Then the multiclass one-versus-one SVM classification, with the Gaussian radial basis function (RBF) kernel, of the HS image was performed. The optimal parameters $\mathrm{C}$ (penalty during the SVM optimization) and $\gamma$ (spread of the RBF kernel) were chosen by fivefold cross validation: $C=128$, $\gamma=2^{-5}$.The results of the pixel-wise classification were combined with the segmentation results using the majority voting approach.

Then, the MSSC-HSEG segmentation of the image was performed, in this experiment, the MSSC-HSEG algorithm has been run until no more merging was possible. Since the image of this urban area contains classes with mostly dissimilar spectral responses, we chose $s_{w g h t}=[0.0,0.2,0.5]$. The proposed Rule-based classification method uses a rule base that combines spectral, textural, geometric, and contextual information.

\begin{tabular}{|c|c|c|c|c|c|}
\hline & $\begin{array}{c}\text { MSSC- } \\
\text { MSF }\end{array}$ & $\begin{array}{c}\text { MSSC- } \\
\text { HSEG+M } \\
\text { V }\end{array}$ & \multicolumn{3}{|c|}{$\begin{array}{c}\text { Proposed rule-based } \\
\text { approach }\end{array}$} \\
\hline$S_{w g h t}$ & & 0.0 & 0.0 & 0.2 & 0.5 \\
\hline $\mathrm{OA}(\%)$ & 94.43 & 96.90 & 98.88 & $\mathbf{9 8 . 9 8}$ & 98.11 \\
\hline $\mathrm{AA}(\%)$ & 95.76 & 96.55 & 97.91 & $\mathbf{9 8 . 1 6}$ & 96.98 \\
\hline$K_{(\%)}$ & 93.94 & 95.82 & 97.82 & $\mathbf{9 8 . 0 4}$ & 97.09 \\
\hline
\end{tabular}

Table 1.Classification Accuracies for the Center of Pavia Image: Overall Accuracy (OA), Average Accuracy (AA), Kappa Coefficient $(\kappa)$, The highest accuracies are bolded in each category. 
In order to compare the results of the proposed method with other advanced Spectral-spatial classification techniques, we have included results obtained using the construction of an MSF from the same sets of markers (MSSC-MSF), and also accuracy of classification by Majority Voting within the neighborhoods defined by Marker-based HSEG segmentation and SVM algorithm (MSSC-HSEG+MV) with the parameter $s_{w g h t}=0.0$.

The accuracies of the obtained maps were assessed by computing the overall accuracy (OA), the average accuracy (AA), and the Kappa coefficient $(\kappa)$ on the available reference data. The obtained results are reported in Table 1. It is clear, the proposed approach, by including the features extracted in the analysis resulted in higher accuracies (up to almost $4 \%$ for MSSC-MSF and 2\% for MSSC-HSEG+MV of OA) than two other approaches.

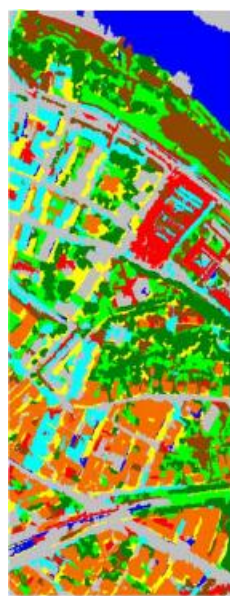

(a)

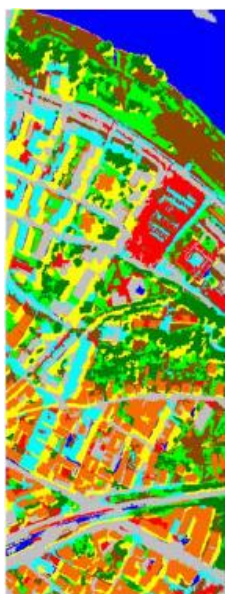

(b)

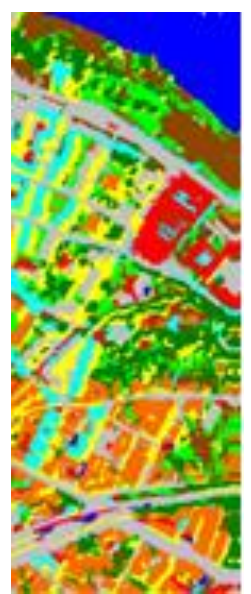

(c)
Figure 3. ROSIS Pavia Center data set. Classification maps obtained by (a) MSSC-MSF, (b) MSSC-HSEG+MV (c) proposed approach

Fig. 3 shows the corresponding classification maps. The Proposed classification map [see Fig. 3(c)] contains much more homogeneous regions when compared with the maps obtained by other spectral-spatial approaches.

From the table can conclude, it is useful to extract spatial features in the MSSC-HSEG algorithm, in order to increase classification accuracy. Moreover the proposed method performs similar with $s_{w g h t}=0.0$ and $s_{w g h t}=0.2$ in terms of classification accuracies. However, classifications accuracies decrease with further increase of the $S_{w g h t}$ value.

\section{CONCLUSIONS}

HS sensors capture images in hundreds of narrow spectral channels. The detailed spectral signatures for each spatial location provide rich information about an image scene, leading to better discrimination between physical materials and objects. Although pixel-wise classification techniques have given high classification accuracies when dealing with HS data, the incorporation of the spatial context into classification procedures yields further improvement of the accuracies.

In this paper, a new method for Rule-based classification of HS images based on MSSC-HSEG segmentation approach has been proposed. First, a marker map is constructed by selecting the pixels assigned by several spectral-spatial classifiers to the same class. This ensures a robust and reliable selection. Then, the MSSC-HSEG algorithm is applied, resulting in a segmentation map. Additional information from image objects also allow us to get shape characteristics and neighborhood relationships is used for the object's classification. However, the success of Rule-based classification approaches is very dependent much on the quality of the image segmentation and the classification method.

The experimental analysis was carried out on a well known HS image acquired on the city of Pavia (Italy). Experimental results, demonstrated that the proposed method improves classification accuracies, when compared with previously proposed classification schemes, and provides classification maps with homogeneous regions. Therefore, it was evident how important the spatial features for classification. Further work is needed to improve the proposed method. It is necessary to take advantage of the available data in order to automate the whole classification process.

\section{ACKNOWLEDGMENT}

The authors would like to thank Prof. P. Gamba, University of Pavia, for providing the ROSIS data set.

\section{REFERENCES}

Beaulieu, J.-M. and Goldberg, M., 1989. Hierarchy in picture segmentation: A stepwise optimization approach. IEEE Trans. Pattern Anal. Machine Intell. vol. 11, no. 2, pp. 150-163.

Benz, U.C., Hofmann, P., Willhauck, G., Lingenfelder, I. and Heynen M., 2004. Multi-resolution, object-oriented fuzzy analysis of remote sensing data for GIS-ready information. ISPRS J. Photogramm, pp. 239-258.

Camps-Valls, G. and Bruzzone, L., 2005a. Kernel-based methods for hyperspectral image classification. IEEE Trans. Geos. and Remote Sens., vol. 43, no. 6, pp. 1351-1362.

Camps-Valls, G., Gomez-Chova, L., Munoz-Mari, J., VilaFrances, J. and Calpe-Maravilla, J., 2006b. Composite kernels for hyperspectral image classification. IEEE Geosci. Remote Sens. Lett., vol. 3, no. 1, pp. 93-97.

Carleer, A. P. and Wolff, E., 2006. Urban land cover multi-level region-based classification of VHR data by selecting relevant features. Int. J. Remote Sens., vol. 27, no. 6, pp. 1035-1051.

Fauvel, M., Chanussot, J., Benediktsson, J. A. and Sveinsson, J. R., 2008. Spectral and spatial classification of hyperspectral data using SVMs and morphological profiles. IEEE Trans. Geosci. Remote Sens., vol. 46, no. 11, pp. 3804-3814.

Goetz, A. F. H., Vane, G., Solomon, J. E. and Rock, B. N., 1985. Imaging spectrometry for Earth remote sensing. Science, 228, pp. 1147-1153.

Gonzalez, R. and Woods, R., 2002. Digital Image Processing. 2nd ed. Englewood Cliffs, NJ: Prentice-Hall.

Green, R. O., Eastwood, M. L., Sarture, C. M., Chrien, T. G., Aronsson, M. and Chippendale, B. J., 1998. Imaging spectroscopy and the airborne visible/infrared imaging spectrometer (AVIRIS). Remote Sensing of Environment, 65, pp. 227-248. 
Jensen, J. R., 2004. Introductory Digital Image Processing-A Remote Sensing Perspective. 3rd ed. Upper Saddle River, NJ: Pearson Prentice-Hall.

Jimenez, L. O., Rivera-Medina, J. L., Rodriguez-Diaz, E., Arzuaga-Cruz, E. and Ramirez- Velez, M., 2005. Integration of spatial and spectral information by means of unsupervised extraction and classification for homogenous objects applied to multispectral and hyperspectral data. IEEE Transactions on Geoscience and Remote Sensing, 43, pp. 844-851.

Kettig, R. L. and Landgrebe, D. A., 1976. Classification of multispectral image data by extraction and classification of homogeneous objects. IEEE Trans. Geosci. Electron., vol. 14, no. 1, pp. 19-26.

Landgrebe, D. A., 2003. Signal theory methods in multispectral remote sensing. Hoboken: John Wiley and Sons.

Li, X., Zhao, S., Rui, Y. and Tang, W., 2007. An object-based classification approach for high-spatial resolution Imagery. Geoinformatics 2007: Remotely Sensed Data and Information, Proc. of SPIE.

Salehi, B., Zhang, Y., Zhong, M. and Dey, V., 2012. A review of the effectiveness of spatial information used in urban land cover classification of VHR imagery. Int. J. GeoInf., pp. 35-51.

Shackelford, A. K. and Davis, C. H., 2003. A combined fuzzy pixel-based and object-based approach for classification of high-resolution multispectral data over urban areas. IEEE Trans. Geosci. Remote Sens., vol. 41, no. 10, pp. 2354-2363.

Soille, P., 2003. Morphological Image Analysis, 2nd ed. Berlin, Germany: Springer-Verlag.

Tarabalka, Y., Benediktsson, J. A. and Chanussot, J., 2009a. Spectral-spatial classification of hyperspectral imagery based on partitional clustering techniques. IEEE Trans. Geos. and Remote Sens., vol. 47, no. 9, pp. 2973-2987.

Tarabalka, Y., Benediktsson, J. A., Chanussot, J. and Tilton, J. C., 2010b. Multiple spectral-spatial classification approach for hyperspectral data. IEEE Trans. Geosci. Remote Sens., vol. 48, no. 11 , pp. $4122-4132$.

Tarabalka, Y., Chanussot, J. and Benediktsson, J. A., 2010c. Segmentation and classification of hyperspectral images using watershed transformation. Pattern Recognit., vol. 43, no. 7, pp. 2367-2379.

Tarabalka, Y., Chanussot, J. and Benediktsson, J. A., 2010d. Segmentation and classification of hyperspectral images using minimum spanning forest grown from automatically selected markers. IEEE Trans. Syst., Man, Cybern. B, Cybern., vol. 40, no. 5, pp. 1267-1279.

Tarabalka, Y., Chanussot, J., Benediktsson, J. A., Angulo, J. and Fauvel, M., 2008e. Segmentation and classification of hyperspectral data using watershed. in Proc. Of IGARSS '08, Boston, USA, pp. III-652 - III-655.

Tilton, J., 1998a. Image segmentation by region growing and spectral clustering with a natural convergence criterion. in Proc. IGARSS'08, vol. 4, pp. 1766-1768.
Tilton, J., 2003b. "Analysis of hierarchically related image segmentations," in Proc. IEEE Workshop Adv. Tech. Anal. Remotely Sensed Data, pp. 60-69.

Tilton, J. C., 2008c. "HSEG/RHSEG, HSEGViewer and HSEGReader User's Manual (Version 1.40) ", Provided With the Evaluation Version of RHSEG. [Online]. Available: http://techtransfer.gsfc.nasa.gov/ft_tech_rhseg.shtm.

Tilton, J. C., 2010d. RHSEG User's Manual: Including HSWO, HSEG, HSEGExtract, HSEGReader and HSEGViewer. version 1.50, via email request to James.C.Tilton@nasa.gov.

Vincent, L. and Soille, P., 1991. Watersheds in digital spaces: an efficient algorithm based on immersion simulations, IEEE Trans. Pattern Analysis and Machine Intel., vol. 13, no. 6, pp. 583-598.

Voirin, Y., 2004. Élaboration d'un Système à Base de Règles Pour l'identification des Zones Perturbées en Milieu Forestier. Ph.D. dissertation, Université de Sherbrooke, Sherbrooke, QC, Canada.

Walker, J.S. and Blaschke, T., 2008. Object-based land cover classification for the Phoenix metropolitan area: optimization vs. transportability. Int. J. Remote Sens., pp. 2021-2040. 